\title{
Decaying Dark Matter at IceCube and its Signature in High-Energy Gamma-Ray Experiments
}

\author{
Barbara Skrzypek, $^{a, *}$ Carlos Argüelles $^{a}$ and Marco Chianese ${ }^{b}$ \\ ${ }^{a}$ Harvard University Physics Department, \\ 17 Oxford St, Cambridge, Massachusetts 02138, United States \\ ' Università degli studi di Napoli "Federico II" and INFN - Sezione di Napoli, \\ Complesso Univ. Monte S. Angelo, I-80126 Napoli, Italy \\ E-mail: bskrzypek@g.harvard.edu, carguelles@fas.harvard.edu, \\ ma.chianese@gmail.com
}

\begin{abstract}
Observations of high-energy astrophysical neutrinos in IceCube have opened the door to multimessenger astronomy, by way of which questions in particle physics could be explored through a combination of IceCube data and optical experiments such as Fermi-LAT. However, the origin of these astrophysical neutrinos is still largely unknown. Among the tensions that still need to be addressed, for example, is the excess of neutrinos observed in the energy range of 40-200 TeV, a contribution that could come from heavy dark matter decay. The dark matter decay hypothesis can be tested through comparisons with gamma-ray data, because a coincident gamma-ray flux is expected to accompany the neutrino flux that IceCube observes. However, gamma-rays become heavily suppressed for sources dominating in particular energy ranges. In the case of the Galactic center, the $\gamma$-sky is partially opaque in the (0.1-10) PeV range. This is due to properties of the traversed medium, which can generally consist of extragalactic background light (EBL), the cosmic microwave background (CMB), and the intergalactic magnetic field. These significantly alter the initial spectrum through intermediate processes such as absorption and Inverse-Compton scattering, giving rise to anisotropy and energy features in the final spectrum that reaches telescopes on Earth. The existence of competing photon background models, moreover, complicates estimates of dark matter constraints. In this presentation, we address these questions by studying the impact that these different models have on indirect measurements of heavy dark matter decay. I present my predictions for galactic, inverse-Compton, and extragalactic gamma-ray spectra undergoing attenuation by different backgrounds.
\end{abstract}

$37^{\text {th }}$ International Cosmic Ray Conference (ICRC 2021)

July 12th - 23rd, 2021

Online - Berlin, Germany

\footnotetext{
*Presenter
} 


\section{Introduction}

IceCube's measurements of ultra-high-energy neutrinos in the TeV-PeV range has opened many possibilities for multimessenger studies of astrophysical sources and astroparticle physics. In particular, the high-energy starting event (HESE) sample, with its sensitivity to the Galactic center and, generally, the entire sky, has revealed an excess of these neutrinos that cannot be explained by a simple, astrophysical, single power-law source [1]. The processes that give rise to these astrophysical sources also produce coincident gamma-rays, such as has been confirmed by multi-messenger observations of the flaring blazar TXS 0506+056. However, the high level of suppression of the gamma-ray flux from potential sources combined with tensions among current IceCube data make it difficult to understand the nature of the source of these ultra-high-energy neutrino sample.

Heavy decaying dark matter has been proposed as a potential source of ultra-high-energy neutrinos [2-4]. As gamma-rays are also produced in dark-matter decays, this scenario has been intensively investigated with gamma-ray telescopes. Present diffuse gamma-rays data have indeed been employed to place strong constraints on the dark-matter lifetime for various decay channels, thus disfavoring the dark-matter hypothesis for IceCube's observations [5-7]. Hence, in order to eventually rule out the decaying dark-matter scenario, it is fundamental to examine the robustness of such limits.

In this work, we elaborate on previous dark-matter decay limits by considering the impact that our choice of extragalactic background light (EBL) model could have on the predicted gamma-ray spectrum from dark-matter. We simulate the propagation of photons using three different models of the EBL distribution, and we compute the final gamma-ray spectrum at Earth. From there, we compare the predicted fluxes to Tibet 2021 [7] data and obtain lower bounds on dark-matter lifetime assuming only a dark-matter contribution. As a result, we find differences between the predicted lower bounds between the different EBL models that could have significant implications for existing limits.

\section{Gamma-ray absorption by EBL and CMB}

Unlike neutrinos, the gamma-ray final-states resulting from dark-matter decay undergo various kinds of interactions as they propagate to Earth. These scattering processes arise from the presence of intermediate magnetic fields as well as background radiation fields such as the Cosmic Microwave Background (CMB). They can already begin to have a substantial effect at energies lower than 100 $\mathrm{TeV}$ for gamma-rays produced outside of the Galaxy. Moreover, there is an additional contribution coming from secondary photons that arise from electron final states by way of mechanisms such as Inverse-Compton (IC) scattering. The resulting final gamma-ray flux that we observe from darkmatter decay, therefore, can be divided into components corresponding to each of these processes.

In general, we can divide the final gamma-ray flux at Earth into a Galactic component and an Extragalactic component, each of which can be further broken down into subcomponents corresponding to the different intermediate processes that contribute to the final spectrum. The subcomponents comprise of primary gamma-rays, produced directly from dark-matter decay, and secondary gamma-rays, produced by secondary processes such as Inverse Compton. 


\subsection{Prompt}

The prompt gamma-ray flux from Galactic dark-matter decay is given by

$$
\frac{d^{2} \phi}{d E d \Omega}=\frac{1}{4 \pi m_{D M} \tau_{D M}} \frac{d N_{\gamma}}{d E} \int d s \operatorname{Att}(E, s) \rho(s, l, b),
$$

where $s, l$ and $b$ are galactic coordinates, which we transform to radial coordinates $r(s, l, b)$ defining the gamma-ray arrival direction, $m_{D M}$ is the dark-matter mass, $\tau_{D M}$ is its lifetime, $\frac{d N}{d E}$ is the differential spectrum of photons produced per dark-matter decay, and the integral is taken over the line-of-sight. We model the dark-matter density profile as an NFW profile, which depends on the Galactic coordinates in the following way:

$$
\begin{gathered}
\rho(r(s, l, b))=\rho_{0}\left[\frac{r}{r_{c}}\left(1+\frac{r}{r_{c}}\right)^{2}\right]^{-1}, \\
r(s, l, b)=\sqrt{s^{2}+R_{s}^{2}-2 s R_{s} \cos b \cos l},
\end{gathered}
$$

where we set $\rho_{0}=0.4 \mathrm{GeV} / \mathrm{cm}^{3}, r_{c}=24 \mathrm{kpc}$, and $R_{S}=8.5 \mathrm{kpc} \mathrm{[2].}$

\subsection{Secondary}

Both Galactic and Extragalactic secondary gamma-rays are also produced by electrons and positrons interacting with background radiation through IC. To obtain the flux of these secondary photons, we generally need to take into account energy losses due to IC and synchrotron radiation.

\subsection{Absorption of Gamma-Rays}

The attenuation factor included in the prompt gamma-ray flux above describes the absorption of gamma-rays as they propagate and interact with background photon radiation through pair production $\gamma \gamma \rightarrow e^{+} e^{-}$. At energies of $\sim \mathrm{PeV}$, the homogonous $\mathrm{CMB}$ is the dominant target for photon attenuation, whereas other targets such as extragalactic background light (EBL) become significant in the lower energy range (state energy) In this study, we are interested in the effects of extragalactic background light on the predicted spectra in particular, which comprises the total stellar light emitted throughout the history of cosmic evolution. This becomes apparent even at the Galactic scale.

Each of these target photon backgrounds has an associated optical depth $\tau_{\gamma \gamma}$ which quantifies the amount of absorption that gamma-rays with a certain energy undergo as they traverse the background radiation. For a given distribution, the optical depth for photons of energy $E_{\gamma}$ coming from a source a distance $L$ away is computed as

$$
\tau_{\gamma \gamma}=L \iint d \epsilon d \theta \sin \theta \sigma_{\gamma \gamma}\left(E_{\gamma}, \epsilon\right) n(\epsilon) \frac{1-\cos \theta}{2},
$$

where $\sigma_{\gamma \gamma}$ is the cross-section for pair-production and $n$ is the differential number density of photons. The optical depth for different backgrounds such as the EBL can be computed in a similar way. To illustrate the impact that the EBL has on the opacity, $e^{-\tau_{\gamma \gamma}}$, the following figures show the opacity for a the CMB alone alongside the opacity when we include a particular modeling of the EBL in addition to the $\mathrm{CMB}$ for photons traveling from a range of different distances (in $\mathrm{kpc}$ ). 

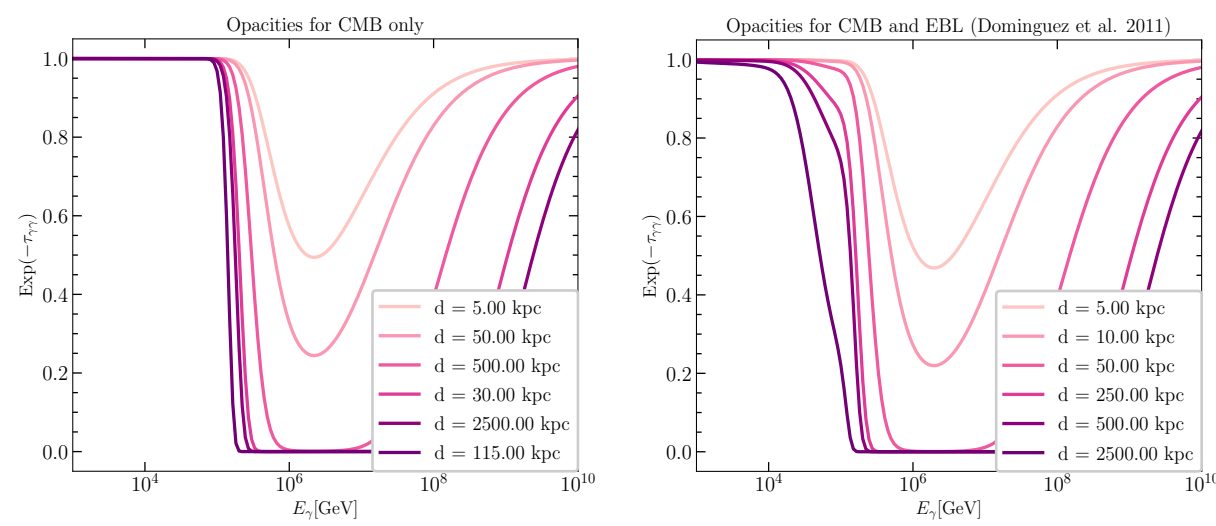

Figure 1: Left: The opacity of photons traversing the CMB as a function of photon energy for several distances. Right: The same plot, but with the Dominguez model of the EBL included as an additional source of background radiation.

To further visualize the effect of the EBL on larger distance scales, the following figure shows the opacities when we consider only the CMB (dashed lines) and when we include the EBL (solid lines). We also show two-dimensional plots, where we see that the EBL begins to have non-negligible effects at energies lower than $10 \mathrm{TeV}$.
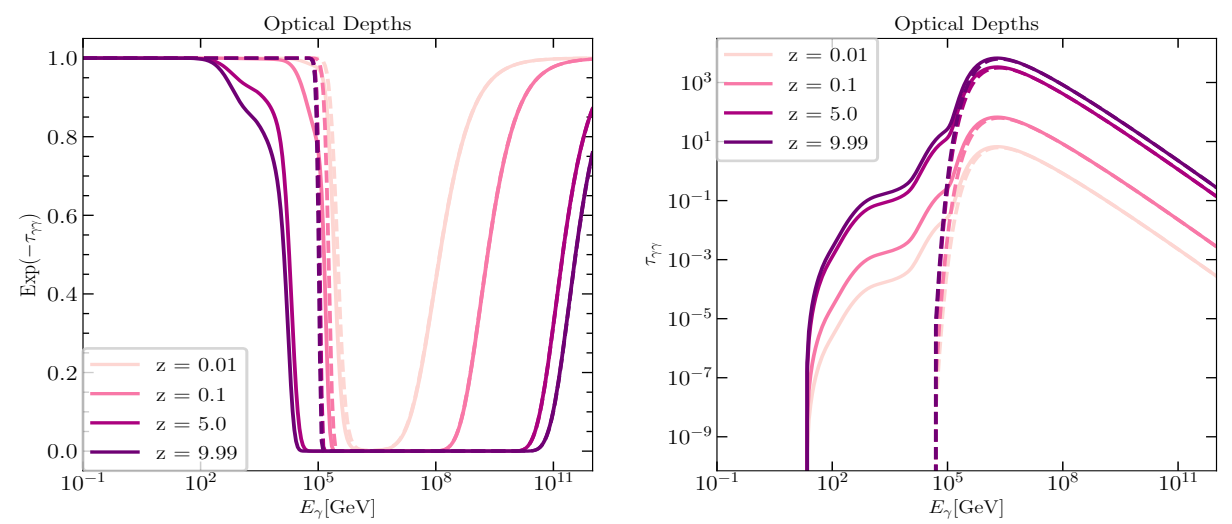

Figure 2: Left: A plot of opacities for photons traversing the CMB (dashed) and the CMB with EBL (solid) for several different redshifts. Right: The optical depth corresponding to the same set of reshifts for the CMB (dashed) and CMB with EBL (solid).

\subsection{EBL Models}

Collective limits obtained from direct and indirect methods show that the EBL has a twopeak structure in the spectral energy distribution (SED). However, the lack of direct knowledge surrounding the structure of EBL has led to the construction of different models for EBL arising from different methods for estimating its structure. Among the prominent models generally considered are:

- Dominguez et al. (2011): An observed evolution model that begins with initial cosmological conditions and evolves forward in time by using semi-analytical models of galaxy 


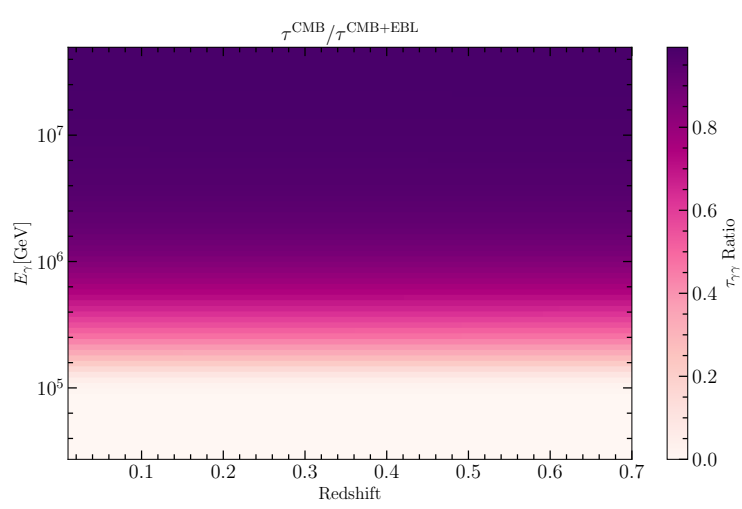

Figure 3: The ratio of the optical depth for CMB only to the optical depth corresponding to the CMB with the EBL as a function of photon energy and reshift.

formation [8],

- Stecker et al. (2016) upper and lower bounds: A backward evolution model that begins with a present-day luminosity function and extrapolates backwards in time [11].

These models predict significantly different photon number densities, and thus also predict different optical depths and final spectra for photons traversing to Earth from dark-matter decay. To obtain more robust limits on dark-matter decay from gamma-ray data, we should take into account this uncertainty in the EBL and check the extent to which it affects the uncertainty on the limits that we place for dark-matter decay.

\section{Simulation Details}

The goal of this work is to understand the impact that the uncertainty in the EBL has on current limits for dark-matter decay. We consider the Galactic contribution, comparing the predictions to Tibet 2021 data [7].

We use CRPropa [9] to propagate photons and electrons over galactic and extragalactic distances. In the former case, we set the galactic magnetic field to be the JF12 field model, and we propagate photons starting from an initial power-law spectrum that follows $\phi \sim\left(E / E_{c}\right)^{-1}$, which we define to be uniformly distributed over distances ranging from 0 to $100 \mathrm{kpc}$ and sampled uniformly on a sphere. CRPropa creates this initial spectrum via a Monte Carlo generation. The minimum and maximum $\left(E_{c}\right)$ energies vary with the dark matter mass that we consider so that $E_{c}=m_{\mathrm{DM}} / 2$ and $E_{\min }=\left(m_{\mathrm{DM}} / 2\right) \times 10^{-6}$. For the propagation, the processes that we enable using CRPropa are: pair production, double pair production, triplet pair production, Inverse-Compton scattering, and synchrotron radiation.

To obtain the expected dark-matter decay spectrum from the output from CRPropa, we apply a reweighting scheme whereupon we group the photons arriving at Earth according to their corresponding unique, Monte-Carlo generated events. The reweighting scheme assigns a new weight to each of these events that depends on that event's initial energy and position, such that the new weights correspond to a dark-matter distribution. Schematically, the end result is that our reweighting scheme takes the final spectrum for an initial power-law distribution and returns the expected 
final spectrum for dark-matter decay of a given dark-matter mass and lifetime. Although we used a specific initial spectrum, this procedure could be generalizes for any arbitrary, well-behaved distribution.

The weight that we assign per each event can be expressed as the product of a position weight, an energy weight, and a prefactor that depends on dark-matter parameters.

\subsection{Position Reweighting}

The position reweighting assigns a multiplicative factor for each event that cancels the Monte Carlo generation weights for a uniformly distributed source and replaces them with weights corresponding to the desired dark-matter profile. Schematically, the position weights can be written as

$$
w=\frac{w_{\text {physical }}}{w_{\text {generated }}}=(\mathrm{DM}(r))\left(\frac{D(r)}{\int_{r_{\text {min }}}^{r_{\max }} D(r)}\right)^{-1}=\mathrm{DM}(r)\left(r_{\text {max }}-r_{\text {min }}\right),
$$

where $D(r)$ is the initial distribution, $\operatorname{DM}(r)$ is the dark-matter distribution, and the denominator of the quantity in parentheses corresponds to an overall normalization included in the generated weights, which for our simulations is a uniform distribution. The physical position weights depend on the profile that we consider. For galactic reweighting, for example, we use $\operatorname{DM}(r)=\rho_{\mathrm{NFW}}(r(s, l, b))$.

\subsection{Energy Reweighting}

In a similar way, the energy reweighting acts as a multiplicative factor that cancels out the Monte-Carlo simulation weights for the power-law spectrum generated by CRPropa and returns a physical weight for each event that corresponds to the expected dark-matter decay energy spectrum. The energy weights can be written as

$$
w=\frac{w_{\text {physical }}}{w_{\text {generated }}}=\left(\phi_{\mathrm{DM}}(E)\right)\left(\frac{\phi(E)}{\int_{E_{\min }}^{E_{\max }} \phi(E)}\right)^{-1}=\phi_{\mathrm{DM}}(E)\left(E \times \ln \left(E_{\max } / E_{\min }\right)\right),
$$

where $\phi(E) \sim E^{-1}$ is the generated initial spectrum from CRPropa. We obtain the dark-matter final state spectra $\phi_{\mathrm{DM}} \propto d N_{\gamma} / d E$ from HDMSpectra [12].

As a final step in the reweighting procedure, we multiply the result by a prefactor that converts the units to those of a flux, introducing the mass and lifetime dependence: $\frac{1}{4 \pi m_{\mathrm{DM}} \tau_{\mathrm{DM}}}$.

\section{Analysis and Results}

In this work, we propagate photons across Galactic distances in the region defined by $b>\left|5^{\circ}\right|$ and $25^{\circ}<l<100^{\circ}$ and apply the reweighting procedure described above to obtain dark-matter spectra corresponding to the channels $\chi \rightarrow b \bar{b}$ and $\chi \rightarrow \tau^{+} \tau^{-}$for various masses and lifetimes, performing this for Dominguez et al. (2011) and the Stecker Upper and Lower (2016) bounds. We then perform a chi-squared likelihood comparison between the predicted distributions and the data points from Tibet 2021, computing the likelihood for different bins in mass and obtaining confidence levels by assuming Wilk's theorem with one degree of freedom. 
The following plots show one example of a benchmark dark-matter scenario with a mass of $m_{\mathrm{DM}}=1.78 \mathrm{PeV}$. At this mass, we observe a difference between three three EBL models which we use in simulations. The plot shows that the two Stecker models can differ from Dominguez up to around 25 percent. We expect this difference to grow even larger for extragalactic distances.
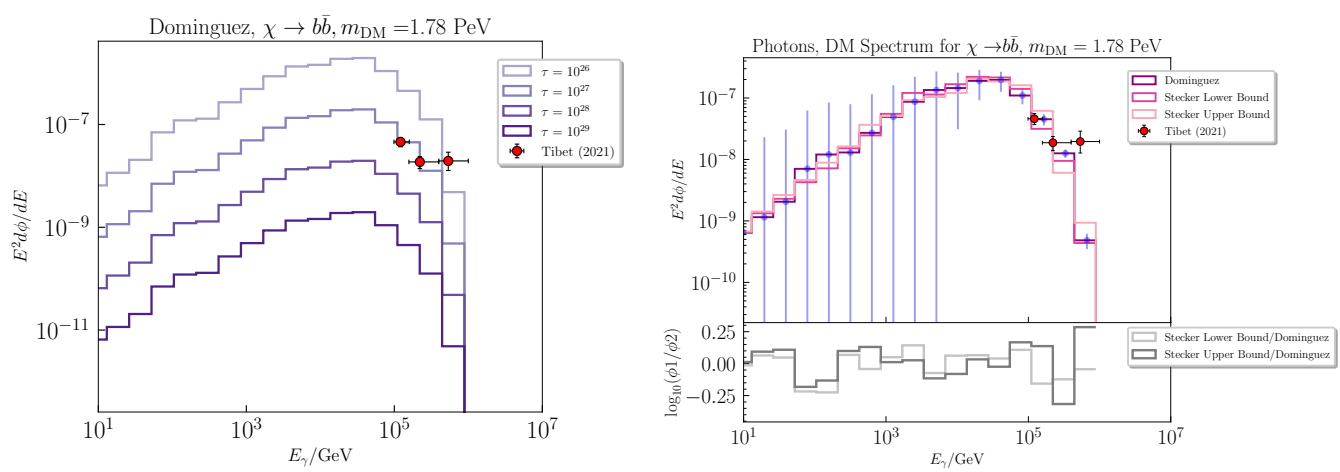

Figure 4: Left: Spectra for the nominal Dominguez et al (2011) model and for a particular dark-matter mass and four lifetimes. Right: Spectra comparing all three EBL models for the same dark matter mass.
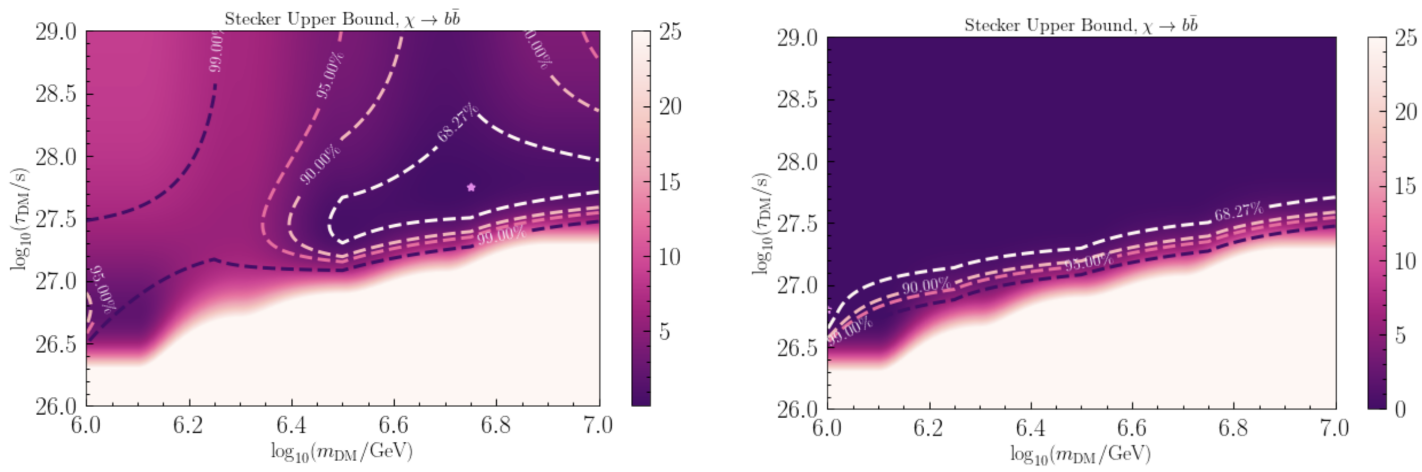

Figure 5: Left: Preferred dark-matter parameter regions for Stecker Upper Bound. Right: Lower bounds on lifetime for Stecker Upper Bound.

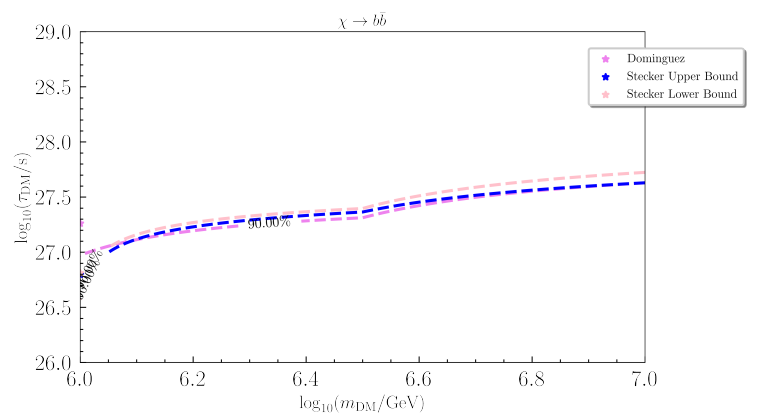

Figure 6: A comparison of the $90 \%$ lower bounds on dark-matter lifetime between the three different EBL models. 


\section{Conclusion}

The propagated gamma-ray spectra from dark-matter for the three EBL models considered here produce considerable differences in both the predicted spectra arriving at Earth as well as lower bounds on lifetime obtained by comparing with Tibet 2021 data. The deviations from the nominal model occur at up to 25 percent for galactic propagation.

As a part of this ongoing work, we plan to complete this analysis for additional dark-matter decay channels and by making similar comparisons with Fermi-LAT diffuse data for extragalactic gamma-rays and IceCube for neutrinos. We expect to see an even larger effect due to EBL for the former. To conclude the analysis, we plan to combine these comparisons to obtain estimates for the uncertainties on current dark-matter constraints. This can be extended to other astrophysical scenarios and processes to understand the effect of the EBL in other studies.

\section{References}

[1] R. Abbasi et al. [IceCube], [arXiv:2011.03545 [astro-ph.HE]].

[2] M. Chianese, D. F. G. Fiorillo, G. Miele, S. Morisi and O. Pisanti, JCAP 11, 046 (2019) doi:10.1088/1475-7516/2019/11/046 [arXiv:1907.11222 [hep-ph]].

[3] B. Feldstein, A. Kusenko, S. Matsumoto and T. T. Yanagida, Phys. Rev. D 88, no.1, 015004 (2013) doi:10.1103/PhysRevD.88.015004 [arXiv:1303.7320 [hep-ph]].

[4] A. Esmaili and P. D. Serpico, JCAP 11, 054 (2013) doi:10.1088/1475-7516/2013/11/054 [arXiv:1308.1105 [hep-ph]].

[5] T. Cohen, K. Murase, N. L. Rodd, B. R. Safdi and Y. Soreq, Phys. Rev. Lett. 119, no.2, 021102 (2017) doi:10.1103/PhysRevLett.119.021102 [arXiv:1612.05638 [hep-ph]].

[6] A. Esmaili and P. D. Serpico, [arXiv:2105.01826 [hep-ph]].

[7] T. N. Maity, A. K. Saha, A. Dubey and R. Laha, [arXiv:2105.05680 [hep-ph]].

[8] A. Esmaili and P. D. Serpico, JCAP 10, 014 (2015) doi:10.1088/1475-7516/2015/10/014 [arXiv:1505.06486 [hep-ph]].

[9] R. Alves Batista, A. Dundovic, M. Erdmann, K. H. Kampert, D. Kuempel, G. Müller, G. Sigl, A. van Vliet, D. Walz and T. Winchen, JCAP 05, 038 (2016) doi:10.1088/14757516/2016/05/038 [arXiv:1603.07142 [astro-ph.IM]].

[10] K. K. Singh and P. J. Meintjes, doi:10.1080/20909977.2020.1743468 [arXiv:2004.01933 [astro-ph.HE]].

[11] F. W. Stecker, S. T. Scully and M. A. Malkan, Astrophys. J. 827, no.1, 6 (2016) [erratum: Astrophys. J. 863, no.1, 112 (2018)] doi:10.3847/0004-637X/827/1/6 [arXiv:1605.01382 [astroph.HE]].

[12] C. W. Bauer, N. L. Rodd and B. R. Webber, JHEP 06, 121 (2021) doi:10.1007/JHEP06(2021)121 [arXiv:2007.15001 [hep-ph]]. 